\title{
Foreign peptide triggers boost in pneumococcal metabolism and growth
}

Fauzy Nasher ${ }^{1,2 \dagger}$, Sunniva Förster ${ }^{1,2,3 \dagger}$, Efe C. Yildirim', Denis Grandgirard', Stephen L. Leib', Manfred Heller ${ }^{4}$ and Lucy J. Hathaway ${ }^{1 *}$

\begin{abstract}
Background: Nonencapsulated Streptococcus pneumoniae bacteria are successful colonizers of the human nasopharynx and often possess genes aliB-like ORF 1 and 2 in place of capsule genes. AliB-like ORF 2 binds peptide FPPQSV, found in Prevotella species, resulting in enhanced colonization. How this response is mediated is so far unknown.

Results: Here we show that the peptide increases expression of genes involved in release of host carbohydrates, carbohydrate uptake and carbohydrate metabolism. In particular, the peptide increased expression of 1,5-anhydroD-fructose reductase, a metabolic enzyme of an alternative starch and glycogen degrading pathway found in many organisms, in both transcriptomic and proteomic data. The peptide enhanced pneumococcal growth giving a competitive advantage to a strain with aliB-like ORF 2, over its mutant lacking the gene.

Possession of aliB-like ORF 2 did not affect release of inflammatory cytokine CXCL8 from epithelial cells in culture and the nonencapsulated wild type strain was not able to establish disease or inflammation in an infant rat model of meningitis.
\end{abstract}

Conclusions: We propose that AliB-like ORF 2 confers an advantage in colonization by enhancing carbohydrate metabolism resulting in a boost in growth. This may explain the widespread presence of aliB-like ORF 2 in the nonencapsulated pneumococcal population in the human nasopharynx.

Keywords: Streptococcus pneumoniae, Peptide, Nonencapsulated, aliB-like ORF 2, Transcriptome, Proteome

\section{Background}

Streptococcus pneumoniae causes several human diseases including bacterial meningitis, pneumonia, sepsis and otitis media but more often inhabits the human nasopharynx without causing disease. It shares this niche with many other organisms and we have previously proposed that pneumococcus senses its bacterial neighbours by recognizing peptide fragments derived from them [1]. Of pneumococcal strains that lack genes to synthesize a polysaccharide capsule, the majority possess in their stead two genes named aliB-like ORF 1 and ORF 2 which we predict encode substrate-binding proteins of an ATP-binding cassette $(\mathrm{ABC})$ transporter $[2,3]$. Nonencapsulated pneumococci which lack capsule

\footnotetext{
* Correspondence: lucy.hathaway@ifik.unibe.ch

${ }^{\dagger}$ Equal contributors

1 Institute for Infectious Diseases, Faculty of Medicine, University of Bern,

Friedbühlstrasse 51, CH-3001 Bern, Switzerland

Full list of author information is available at the end of the article
}

genes but have aliB-like ORF 1 and ORF 2 are commonly of multilocus sequence types 344 or 448 and close relatives, which make up a separate phylogenetic group that clusters away from the majority of encapsulated pneumococci [3-5]. As well as being efficient colonizers of the nasophynyx, these nonencapsulated strains, particularly ST448, are associated with conjunctivitis and have been proposed to be adapted to these niches by distinct virulence factors and metabolic features which differ from those of encapsulated pneumococci [6]. The aliB-like ORF 1 and ORF 2 genes are also found in strains of the commensal streptococci S. mitis, S. oralis and S. pseudopneumoniae [7-9]. By expressing AliB-like ORF 1 and ORF 2 proteins, we have previously shown that they bind to peptides SETTFGRDFN (found in the 50S ribsosomal subunit protein L4 of Enterobacteriaceae) and FPPQSV (found in proteins of Prevotella species) respectively. Binding of the ORF 1 ligand was associated with upregulation of competence for genetic transformation whereas 
binding of ORF 2 ligand aided the pneumococcal colonization of the nasopharynx [1]. Understanding the communication that occurs between bacterial species in their natural environment may provide novel targets with which to interfere.

Here, we investigated how binding of AliB-like ORF 2 to its ligand causes phenotypic changes to the pneumococcus. We determined the changes caused by the peptide ligand to the pneumococcal transcriptome and proteome and the effect on growth in vitro. We also determined the effect of pneumococci with and without AliB-like ORF 2 on host cells in vitro and in an infant rat model of meningitis.

\section{Methods}

\section{Ethics statement}

All animal studies were approved by the Animal Care and Experimentation Committee of the Canton of Bern, Switzerland (licence BE76/14) and followed the Swiss national guidelines for the performance of animal experiments.

\section{Bacterial culture and strains}

Bacteria were stored and cultured as described previously [1] i.e., storage using Protect bacterial preservers (Technical Service Consultants, Heywood, U.K.) at $-80{ }^{\circ} \mathrm{C}$. To grow the pneumococci they were first streaked onto Columbia sheep blood agar (CSBA) plates and then incubated at $37^{\circ} \mathrm{C}, 5 \% \mathrm{CO}_{2}$ overnight. Cultures of 3 to 10 colonies were next prepared in $5 \mathrm{ml}$ brain heart infusion (BHI) broth (Becton Dickinson and Company, le Pont de Claix, France) containing 5\% fetal calf serum (FCS) (Biochrom KG, Berlin, Germany) for overnight culture.

The Swiss nonencapsulated nasopharyngeal pneumococcal isolate 110.58 of multilocus sequence type (MLST) ST344 [2], and the construction of its mutants in which one or both of its aliB-like ORFs have been inactivated to give mutants $\triangle \mathrm{ORF} 2$ and $\triangle \mathrm{ORF} 1+2$ has been previously described $[1,2]$.

\section{Preparation of bacteria and measurement of growth}

Following overnight growth on CSBA plates at $37^{\circ} \mathrm{C}, 5 \%$ $\mathrm{CO}_{2}$, the bacteria were subcultured in $\mathrm{BHI}+\mathrm{FCS}$ medium, grown to $\mathrm{OD}_{600 \mathrm{~nm}} 0.5$ and subsequently centrifuged at $3000 \mathrm{~g}$ for $5 \mathrm{~min}$ before resuspension in chemically defined CDM medium which contains $5.5 \mathrm{mM}$ glucose [10]. Growth was monitored as described previously [11] in sterile flat-bottomed 96-well microtitre plates (Nunclon Surface, Nunc, Denmark) based on the method of Brewster [12] as follows: $200 \mu \mathrm{l}$ bacteria culture was incubated per well at $37{ }^{\circ} \mathrm{C}$ and $\mathrm{OD}_{450 \mathrm{~nm}}$ was measured at $30 \mathrm{~min}$ intervals by a VERSAmax microplate reader (Molecular Devices) over $20 \mathrm{~h}$ with $5 \mathrm{~s}$ of automatic shaking preceding each reading. Condensation was prevented by pre-treating the plate lids with $3 \mathrm{ml} 0$. 05\% Triton X-100 in 20\% ethanol [11].

\section{Growth competition assay}

Wild type strain 110.58 and mutant $\triangle \mathrm{ORF} 2$ were streaked onto CSBA plates and incubated at $37{ }^{\circ} \mathrm{C}$ in a $5 \% \mathrm{CO}_{2}$-enriched atmosphere overnight then subcultured in $\mathrm{BHI}+\mathrm{FCS}$ medium to $\mathrm{OD}_{600 \mathrm{~nm}} 0.5$, centrifuged at $3000 \mathrm{~g}$ for $5 \mathrm{~min}$ and resuspended in chemically defined CDM medium. $250 \mu \mathrm{l}$ of each bacterial culture was transferred to $4.5 \mathrm{ml} \mathrm{CDM}$ pre-warmed to $37{ }^{\circ} \mathrm{C}$ and AliB-like ORF 2 ligand FPPQSV (synthesized by PolyPeptide Group, Strasbourg, France) was added to a final concentration of $0.07 \mathrm{mg} / \mathrm{ml}(104 \mu \mathrm{M})$ and the culture incubated to an $\mathrm{OD}_{600 \mathrm{~nm}}$ 0.3. Serial dilutions in PBS were plated onto CSBA plates with and without $3 \mu \mathrm{g} / \mathrm{ml}$ chloramphenicol to differentiate between wild type (chloramphenicol susceptible) and mutant $\triangle$ ORF 2 (resistant) strains. After overnight incubation, the number of colonies was counted and the colony forming units (CFU) for each strain calculated. (CFU for mutant was calculated from the number of colonies on the chloramphenicol plates, CFU for wild type was calculated from the number of colonies on the CSBA plates minus the number on the chloramphenicol plates).

Gene expression analysis by RNA-Seq and real-time RT-PCR RNA-Seq was used to identify differentially expressed genes between wild type and $\triangle \mathrm{ORF} 2$ mutant as well as wild type compared to wild type exposed to the peptide ligand and the $\triangle$ ORF 2 mutant compared to ORF 2 treated with the ligand.

110.58 and $\triangle$ ORF 2 were plated out on CSBA plates and incubated at $37{ }^{\circ} \mathrm{C}, 5 \% \mathrm{CO}_{2}$ overnight, colonies picked and cultured overnight in $5 \mathrm{ml} \mathrm{BHI}+$ FCS as described above until $\mathrm{OD}_{600 \mathrm{~nm}}=0.4$ then centrifuged at $2000 \mathrm{~g}$ for $5 \mathrm{~min}$ and the pellet resuspended in CDM. The centrifugation was repeated and the pellet resuspended in $5 \mathrm{ml}$ CDM. This $5 \mathrm{ml}$ bacterial suspension was added to $10 \mathrm{ml}$ CDM. For each strain, at exactly $\mathrm{OD}_{600 \mathrm{~nm}}=0.2$ the culture was split into two tubes, each containing $5 \mathrm{ml}$. To one tube for each strain the peptide FPPQSV was added to give a final concentration of 0 . $07 \mathrm{mg} / \mathrm{ml}$, mixed and all tubes incubated at $37{ }^{\circ} \mathrm{C}$ for exactly $15 \mathrm{~min}$. Transcription was stopped by adding RNAprotect (Qiagen) and RNA extracted as described previously [13]. RNA was isolated from three separate experiments performed on different days for RNA-Seq and from a further three separate experiments performed on different days for real-time RT-PCR.

For RNA-Seq, ribosomal RNA was depleted using Ribominus (Invitrogen) and then the RNA purified using RNA Clean \& Concentration ${ }^{\mathrm{TM}}-5$ kit (Zymo Research) according to the manufacturers' instructions, eluting in 
$6 \mu$ l. Libraries were prepared using TruSeq ${ }^{\circ}$ Stranded mRNA (Illumina). $13 \mu$ l Fragment, Prime, Finish Mix was added to $5 \mu \mathrm{l}$ of the mRNA. Fragmentation was performed for $40 \mathrm{~s}$ at $94{ }^{\circ} \mathrm{C}$. Raw reads were obtained from an Illumina $3000 \mathrm{Hi}$-seq paired end sequencing platform. Reads were mapped to Streptococcus pneumoniae strain NT_110_58 assembly gca 000817005 ASM81700v1). Preprocessing and adapter triming was done using the Trimommatic tool (0.35) [14]. Alignments were performed with Bowtie (bowtie2-2.2.8) [15]. Cufflinks (cufflinks-2.2.1) and Cuffdiff were used to count reads and calculate differential gene expression [16]. Quality control and coverage were examined using FastQC (0.11.5) and Qualimap 2.2.1 [17]. The sequencing and coverage was on average 3557 times the genome size (ranging between 543 and 5220). The mean length of sequenced fragments was $141 \mathrm{bp}$. Mapping to the reference genome was above $99.09 \%$ for all samples.

Expression of genes of interest identified by RNA-Seq was quantified by real-time RT-PCR as described previously [13] and normalized against $16 \mathrm{~S}$ using the following primers and probes: $16 \mathrm{~S}$ forward primer 5'GACGATACATAGCCGACCTGAGA-3'; reverse primer 5'-GTAGGAGTCTGGGCCGTGTCT-3'; probe 6carboxyfluorescein (6-FAM)-CCAGTGTGGCCGATCminor groove binder (MGB); afr forward primer $5^{\prime}$ - TC TTCATCACCGAAATGTTCACCTT-3'; reverse primer 5' - ATGCCTGAAACTGTAACCATGACA-3'; probe, (6FAM)- ATGGGCCACATTTCCA-(MGB); adhE forward primer 5'-GAAGGAAGTTTCATCCATTGCAT GT-3'; reverse primer 5'-ACGTTAGTGCCATTAA CCTCTTGAA-3'; probe (6-FAM)-CCGTCTTCCGAC TTTT-(MGB); nanA_3 forward primer 5'-CACCACTTCACCAGCAGATGTATAA-3'; reverse primer 5'-GAG ACTAAAGTTCCAATAACGACTGGTT-3'; probe (6FAM)-CACGCACCATTTTCTT-(MGB); nanB forward primer 5'-GTTAACCCAACTTTAGCAATGGCAAT-3'; reverse primer 5'-GGAGCAATCATGTCGAGACTACT-3'; probe (6-FAM)-TTCCCACCAATTTTG(MGB); yesO_2 forward primer 5'-ACTTGGTTAGGA AGAGCTGTACTGA-3'; reverse primer 5'-GAATGA CTTCTATACTAAATGGACTACAGGTT-3'; probe (6-FAM)-ATCTGGCACATTTCC-(MGB); $y c j O$ forward primer 5'-GAAGTGGCGTACTCTGTGAAGA3'; reverse primer 5'-TGGACCGTTTTCTCATTAG TTGGT-3'; probe (6-FAM)-AAGCCAATACAAACC C-(MGB).

\section{Proteomic analysis}

Sample processing, LC-MS/MS and data interpretation was essentially done as described previously [18] with the following minor changes. LC-MS/MS analysis was carried out on an Ultimate3000 nanoLC coupled to an Orbitrap Fusion Lumos instrument (ThermoFisher
Scientific) acquiring full MS scans in the $\mathrm{m} / \mathrm{z}$ range $400-1400$ in the orbitrap at resolution $120^{\prime} 000$ with AGC set to $4 \mathrm{e} 5$ and maximal ion injection time of 50 ms. Peptide precursors with charge 2-8 were fragmented once in the iontrap then excluded for 30s. The iontrap setting were data-dependent MS2 cycle time of $3 \mathrm{~s}$, isolation width of $1.6 \mathrm{~m} / \mathrm{z}$, fragmentation $\mathrm{HCD}$ mode with $30 \%$ normalized collision energy, AGC of 1e4 with maximal ion injection time of $35 \mathrm{~ms}$. The LC-MS/ MS data was processed with MaxQuant (version 1.5.4.1) using default settings for peak detection, strict trypsin cleavage rule allowing for up to three missed cleavages, variable oxidation on methionine and acetylation of protein $\mathrm{N}$-termini with strict carbamidomethylation of cysteines. Match between runs was activated with a retention time window of $0.7 \mathrm{~min}$. The fragment spectra were interpreted with the Streptococcus pneumoniae ensemble database (version gca_000817005_ASM81700v1). For data analysis, peptide feature intensities reported in the evidence file were median normalized and missing values were imputed from the low end of the LOG2 transformed intensity distribution of each LC-MS/MS run using Perseus (version 1.5.5.3) as suggested by Lazar et al. [19]. The three most intense peptide feature intensities were summed for the individual protein group intensity.

\section{Detroit cell culture and CXCL8 (IL-8) cytokine assay}

CXCL8 levels were measured as described previously [20]. The human pharyngeal epithelial cell line Detroit 562 (ATCC CCL-138) was cultured in complete medium at $37{ }^{\circ} \mathrm{C}$ at $5 \% \mathrm{CO}_{2}$. This was composed of Minimum Essential Media (MEM) with 10\% heat-inactivated fetal calf serum (FCS), $2 \mathrm{mM}$ of L-glutamine, $1 \%$ sodium bicarbonate, $1 \times$ MEM non-essential amino acid solution, $1 \mathrm{mM}$ sodium pyruvate (all from Gibco, Life Technologies, Switzerland), $100 \mu \mathrm{g} / \mathrm{ml}$ streptomycin and $100 \mathrm{U} /$ $\mathrm{ml}$ penicillin. 0.05\% Trypsin-EDTA (Gibco, Switzerland) was used to harvest the cells when they reached 70-90\% confluence. The production of CXCL8 in response to exposure to S. pneumoniae was determined as described previously $[20]$ as follows.

$3 \times 10^{5}$ Detroit cells in $1 \mathrm{ml} \mathrm{MEM} \mathrm{without} \mathrm{antibiotics,}$ was added per well of a 24-well plate (TPP tissue culture plates, Sigma-Aldrich,) which was then incubated overnight at $37{ }^{\circ} \mathrm{C}, 5 \% \mathrm{CO}_{2}$. Integrity of the monolayer on the following day was checked by microscopy. The medium was replaced in each well by $0.5 \mathrm{ml}$ MEM without FCS or antibiotics. A suspension of bacteria (strain 110.58 or one of its mutants $\triangle$ ORF $1+2$ or $\triangle$ ORF 2 ) of approximately $6 \times 10^{6} \mathrm{CFU} / \mathrm{ml}$ was made to give an estimated MOI of 10 . Serial dilutions of the suspension were made and plated out to enable accurate quantification of CFU $/ \mathrm{ml}$, and therefore the MOI. $0.5 \mathrm{ml}$ 
MEM containing the bacteria $\left(3 \times 10^{6} \mathrm{CFU}\right)$ was added per well. For those wells which received ORF2 ligand peptide FPPQSV, it was added to give a concentration of $0.07 \mathrm{mg} / \mathrm{ml}$. The plate was centrifuged at $120 \mathrm{~g}$ for 3 min at $25{ }^{\circ} \mathrm{C}$ and then incubated at $37{ }^{\circ} \mathrm{C}, 5 \% \mathrm{CO}_{2}$. After an incubation of a total of $24 \mathrm{~h}$ at $37{ }^{\circ} \mathrm{C}, 5 \% \mathrm{CO}_{2}$, the supernatant was collected in $1.5 \mathrm{ml}$ tubes, spun at $20000 \mathrm{~g}$ for $3 \mathrm{~min}$ at room temperature and the supernatant collected and stored at $-80{ }^{\circ} \mathrm{C}$ for later analysis. CXCL8 concentrations were measured by ELISA (R\&D systems ELISA kits, Abingdon, United Kingdom). Experiments were performed in triplicate on three different days.

\section{Infant rat model of pneumococcal meningitis}

A well-established infant rat model of bacterial meningitis [21] adapted to strains not previously passaged in animals [22] was used as follows. As in our previous publication [22] a litter of 12 nursing Wistar rats with their dam was obtained from Charles River (Sulzfeld, Germany) and given 5 days to acclimatize. At 11 days of age rats weighing $24.1 \pm 2$ g were infected intracisternally with $10 \mu \mathrm{l} 0.85 \% \mathrm{NaCl}$ containing either $1.25 \times 10^{7}$ colony forming units $(\mathrm{CFU}) / \mathrm{ml}$ live $S$. pneumoniae strain 110.58 or $5 \times 10^{6}$ colony forming units $(\mathrm{CFU}) / \mathrm{ml}$ live $S$. pneumoniae mutant $\triangle \mathrm{ORF} 1+2$. Samples of cerebrospinal fluid (CSF) were taken at $21 \mathrm{~h}$ post infection (hpi) for quantitative analysis of bacterial cultures to determine whether there was any bacterial meningitis. At 21 and 29 hpi the antibiotic ceftriaxone (Rocephine, Roche Pharma, Basel, Switzerland; $2 \times$ $100 \mathrm{mg} / \mathrm{kg} / \mathrm{d}$ i.p.) was administered. Disease severity was assessed and CSF sampled as described previously for determination of cytokine concentrations at 21 and 45 hpi [22]. Animals were sacrificed with an overdose of pentobarbital $(150 \mathrm{mg} / \mathrm{kg})$ at $45 \mathrm{hpi}$ and perfused with 4\% paraformaldehyde (PFA) in PBS and then the brains were removed and fixed in PFA for histological analysis. The concentration of the cytokines IL-6, IL-1 $\beta$, TNF $\alpha$, IL-10, IFN- $\gamma$ in the CSF samples was determined using the microsphere-based multiplex assays (MILLIPLEX ${ }^{\circ}$ MAP Kit, Rat Cytokine/Chemokine Magnetic Bead Panel, Millipore Corporation, Billerica, MA, USA) as described previously [23]. Cortical damage and apoptosis in the hippocampus was assessed as previously described in animals sacrificed at $45 \mathrm{hpi}[21,23]$.

\section{Statistical analysis}

Statistical analysis of RNA-Seq data was done as previously described [16]. FDR was taken into account with Benjamini-Hochberg correction for multiple testing. Differentially expressed genes were considered significant when the $p$-value of three independent biological experiments was below 0.5 .
For proteomics data analysis $t$-tests were performed within Perseus, including a permutation-based false discovery rate estimation (cutoff at $1 \%$ and using SO function set at 0.5 ) to correct for multiple testing.

For other experiments student $t$-tests were performed to obtain $p$-values using the software GraphPad Prism (Version 7, GraphPad Software, Inc.).

\section{Results}

\section{AliB-like ORF 2 peptide ligand FPPQSV affects gene expression}

Transcriptome analysis by RNA-Seq was performed for wild type nonencapsulated pneumococcal strain 110.58, which possesses aliB-like ORF 1 and ORF 2 in place of capsule genes, and its mutant lacking aliB-like ORF 2 expression $(\triangle \mathrm{ORF} 2)$ in the presence and absence of AliB-like ORF 2 peptide ligand FPPQSV. There was a modest difference in gene expression between the wild type strain 110.58 and its mutant $\triangle \mathrm{ORF} 2$ without treatment but exposure to the ORF 2 ligand FPPQSV caused significant changes in gene expression in strain 110.58 and in mutant $\triangle$ ORF 2 (Additional file 1: Figure S1 and Additional files 2: Table S1, Additional file 3: Table S2, Additional file 4: Table S3, Additional file 5: Table S4). The top twenty most upregulated genes in the wild type following exposure to the ORF 2 ligand peptide are shown in Table 1. This table was produced following exclusion of those genes for which the wild type treated with the peptide gave expression values of less than 20 as even such small values could give statistically significant results if the control group had an even smaller value. Genes for which the change in expression was not significant were also excluded. Of these 20 most upregulated genes, at least 12 are associated with carbohydrate metabolism or uptake (indicated in Table 1 in bold).

Kegg pathways with genes that were significantly overrepresented were "ribosomal proteins", "alanine, aspartate and glutamate" and "purine metabolism". Branched chain amino acid transporters (liv operon) were also differentially regulated (Additional file 1: Figure S2).

To verify the RNA-Seq results, we performed realtime RT-PCR on several genes as follows. The first was afr which encodes 1,5-anhydro-D-fructose reductase. We also determined expression of $a d h E$ which encodes a bifunctional acetaldehyde-alcohol dehydrogenase, primarily a fermentative enzyme. AdhE has been proposed to act as a pneumococcal virulence factor by increasing expression of pneumolysin and therefore haemolytic activity [24]. We also tested by real-time RT-PCR the expression of genes nanA_3 and nanB which encode sialidases as well as yes $O_{2} 2$, encoding a substrate binding protein, and $y c j O$, encoding a permease, of an $\mathrm{ABC}$ transporter for carbohydrate (Fig. 1). nanA_3, nanB, 
Table 1 Summary of top 20 genes with expression upregulated in wild type strain 110.58 by exposure to ORF 2 ligand peptide FPPQSV

\begin{tabular}{|c|c|c|c|c|}
\hline Gene number & Gene name & Function & Ratio $^{a}$ & q \\
\hline SpnNT_02141 & & tRNA-Phe & infinite $^{b}$ & 0.03 \\
\hline SpnNT_02309 & & tRNA-Asn & infinite $^{b}$ & 0.03 \\
\hline SpnNT_01742 & afr & 1,5 -anhydro- $D$-fructose reductase & 20.2 & 0.001 \\
\hline SpnNT_01743 & $\operatorname{nan} B$ & Sialidase & 11.9 & 0.001 \\
\hline SpnNT_01746 & yesO_2 & Putative $A B C$ transporter for carbohydrate & 10.1 & 0.001 \\
\hline SpnNT_01745 & yсjO & Putative $A B C$ transporter for carbohydrate & 7.5 & 0.001 \\
\hline SpnNT_01744 & ycjP & Putative $A B C$ transporter for carbohydrate & 7.5 & 0.001 \\
\hline SpnNT_00088 & sorB_1 & Sorbose-specific phosphotransferase enzyme IIB component & 6.6 & 0.04 \\
\hline SpnNT_01738 & yeso_1 & Putative $A B C$ transporter for carbohydrate & 6.3 & 0.001 \\
\hline SpnNT_01747 & tabA & Toxin-antitoxin biofilm protein TabA & 6.2 & 0.001 \\
\hline SpnNT_01751 & nanA_3 & Sialidase & 5.3 & 0.001 \\
\hline SpnNT_01537 & & CsbD-like protein, general stress response protein & 4.6 & 0.001 \\
\hline SpnNT_01944 & & tRNA-Leu & 4.5 & 0.005 \\
\hline SpnNT_00467 & grpE & HSP-70 cofactor, heat shock protein & 4.4 & 0.001 \\
\hline SpnNT_01021 & pyrp & Uracil transporter & 4.2 & 0.001 \\
\hline SpnNT_00091 & $\operatorname{man} X \_1$ & EIIAB-Man, carbohydrate transport & 4.1 & 0.001 \\
\hline SpnNT_00092 & agas & Putative tagatose-6-phosphate ketose/aldose isomerase & 4.1 & 0.001 \\
\hline SpnNT_00089 & agaC_1 & PTS system N-acetylgalactosamine-specific EIIC component 1 & 4.1 & 0.001 \\
\hline SpnNT_02080 & adhE & Aldehyde-alcohol dehydrogenase & 4.1 & 0.001 \\
\hline SpnNT_01032 & pyrR & Pyrimidine operon attenuation protein/uracil phosphoribosyltransferase & 3.8 & 0.001 \\
\hline
\end{tabular}

Genes names in bold indicate an association with carbohydrate metabolism or uptake. For the complete dataset for the wild type and $\Delta$ ORF 2 mutant see Additional files 2: Table S1, Additional file 3: Table S2, Additional file 4: Table S3, Additional file 5: Table S4

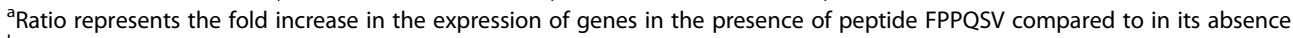

${ }^{b}$ Expression in the absence of peptide was 0 so fold increase in the presence of peptide could not be calculated

yesO_2 and $y c j O$ are located in close proximity to each other in the genome. According to RNA-Seq results in Table 1 afr had an increase in expression of 20.2-fold and $a d h E$ of 4.1 -fold in the wild type strain when it was exposed to the peptide. Real-time RT-PCR indicated a significant 6-fold increase in the expression of afr and 14-fold expression in $a d h E$ when exposed to the peptide (Fig. 1a and b), in line with the RNA-Seq result. Expression of both genes was also increased in the $\triangle \mathrm{ORF} 2$ mutant by exposure to the peptide but to a lesser extent (Fig. 1a and b). The two sialidases (nanA_3 and nanB) also showed upregulation in response to the ORF 2 peptide in the RT-PCR experiments (Fig. 1c and d) which was significant in the wild type strain but not the $\triangle$ ORF $2 \mathrm{mu}$ tant. For the carbohydrate $\mathrm{ABC}$ transporter genes, both the substrate binding protein $\left(y e s O \_2\right)$ and permease $(y c j O)$ were upregulated in both the wild type and the mutant in response to the peptide (Fig. 1e and f). This upregulation was statistically significant for the mutant only for yesO_2 and for both the wild type and mutant for $y c j O$ in contrast to the RNA-Seq results where upregulation was significant for both yesO_2 and $y c j O$ for the wild type but not the mutant (Additional file 2: Table S1).
Proteomic data indicate peptide activates metabolism

To determine whether changes seen in gene transcription are translated into changes at the protein level, proteomic analysis was performed by LC-MS/MS on wild type strain 110.58 and mutant $\triangle \mathrm{ORF} 2$ in the presence and absence of AliB-like ORF 2 peptide ligand FPPQSV. Table 2 shows all proteins significantly upregulated in 110.58 following exposure to peptide FPPQSV (see Additional file 6: Table S5, Additional file 7: Table S6, Additional file 8: Table S7, Additional file 9: Table S8 for all data). The proteomic and RNA-Seq datasets were comparable (Additional file 1: Figure S3) with the top of the list of proteins being Afr (1,5- anhydro-D-fructose reductase), supporting the RNA expression data that the peptide may boost carbohydrate metabolism. In addition, the uracil transporter PyrP was also found to be upregulated by 3.8 fold in both the RNASeq data (Table 1) and the proteomics data (Table 2). NanA_3 and NanB, which were upregulated by ORF 2 peptide in the RNA-Seq and real-time RT-PCR data were not found in the proteomics data.

Since RNA-Seq and proteomic data indicated a boost in metabolism, we next looked at the effect of ORF 2 peptide on pneumococcal growth. 

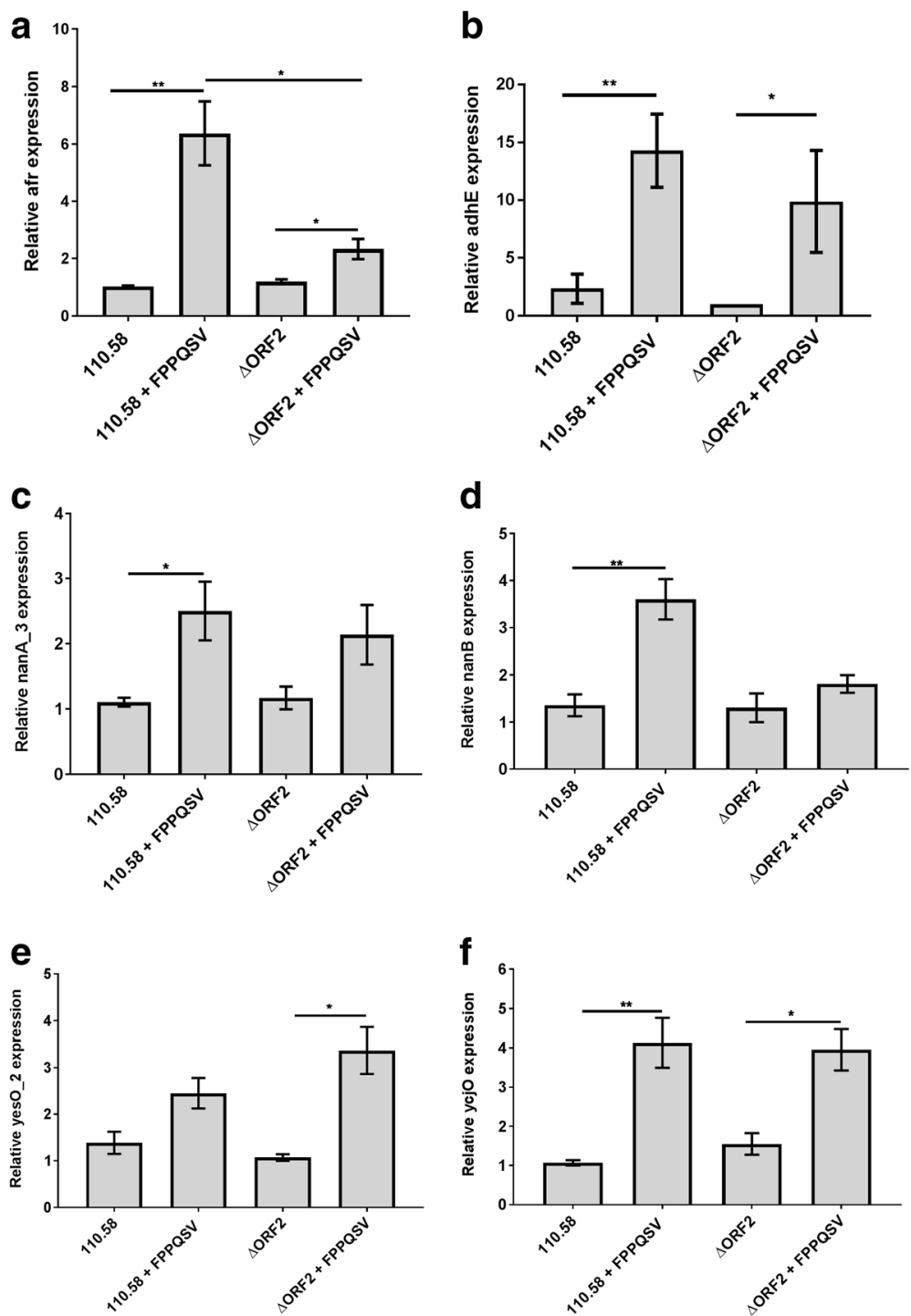

Fig. 1 Relative gene expression. a afr, $\mathbf{b}$ adhE, c nan_A3, $\mathbf{d}$ nanB, e yesO_2 and $\mathbf{f} y c j \mathrm{O}$ expression was determined by real-time RT-PCR and is displayed relative to the value of the lowest expression, after normalization using $16 \mathrm{~S}$ RNA gene expression. The values are the means of four (afr) or three (all other genes) separate experiments. Error bars indicate standard error., ${ }^{*} p \leq 0.05,{ }^{*} p \leq 0.01$

\section{ORF 2 peptide boosts growth}

In CDM, wild type strain 110.58 and mutant $\triangle \mathrm{ORF} 2$ had a similar pattern of growth. Addition of ORF 2 peptide ligand FPPQSV reduced the lag phase of growth and increased the maximum OD for both, but to a greater degree for 110.58 (Fig. 2). For clarity, no error bars are shown in Fig. 2. Growth curves were performed with 4 different doses of ORF 2 peptide, plus no peptide controls, in three independent experiments on three different days. The mean values for all doses, with error bars showing standard error, are shown in Additional file 1: Figure S4A. To confirm that the effect is specific to ORF 2 peptide, growth curves with and without a control peptide of sequence LRRASLG are shown in Additional file 1: Figure S4B and indicate no difference in growth between wildtype and mutant $\triangle$ ORF 2.

To determine whether AliB-like ORF 2 gives a competitive advantage to bacteria which possess it when ORF 2 peptide is present, we next cultured the wildtype strain 110.58 and its $\triangle \mathrm{ORF} 2$ mutant together. Figure 3 shows that when equal numbers of the two strains were mixed together the wild type 110.58, which has the ORF 2 binding protein, has a competitive advantage and that this advantage is significantly greater in the presence of the peptide. 
Table 2 Proteins upregulated in wildtype strain 110.58 by exposure to ORF 2 ligand peptide FPPQSV

\begin{tabular}{|c|c|c|c|c|}
\hline Protein number & Protein name & Function & Ratio $^{a}$ & q \\
\hline AJD72675 & Afr & 1,5-anhydro-D-fructose reductase & infinite $^{b}$ & 0 \\
\hline AJD71621 & DivlB & Cell division protein DivlB & 7.1 & 0.004 \\
\hline AJD71961 & PyrP & Uracil transporter & 3.8 & 0.01 \\
\hline AJD71716 & & ASCH domain protein, Predicted RNA-binding protein YhfF, could regulate translation & 3.8 & 0.01 \\
\hline AJD73085 & & $\begin{array}{l}\text { Hypothetical protein, N-ethylammeline chlorohydrolase, hydrolase activity, } \\
\text { acting on carbon-nitrogen (but not peptide) bonds }\end{array}$ & 3.8 & 0.01 \\
\hline AJD71419 & CitS & Sensor protein & 2.6 & 0.01 \\
\hline AJD71122 & RibE & Riboflavin synthase & 2.3 & 0.01 \\
\hline AJD71434 & $\operatorname{trmB}$ & tRNA (guanine-N(7)-)-methyltransferase & 2.3 & 0.01 \\
\hline AJD71098 & & Hypothetical protein, transcriptional activator, Rgg/GadR/MutR family & 1.7 & 0.02 \\
\hline AJD71572 & & Hypothetical protein & 1.4 & 0.05 \\
\hline AJD71120 & $\mathrm{RibH}$ & 6,7-dimethyl-8-ribityllumazine synthase & 1.4 & 0.04 \\
\hline
\end{tabular}

For the complete dataset for the wild type and $\triangle$ ORF 2 mutant see Additional file 6: Table S5, Additional file 7: Table S6, Additional file 8: Table S7, Additional file 9: Table S8

${ }^{a}$ Ratio represents the fold increase in the protein in the presence of peptide FPPQSV compared to in its absence

${ }^{\mathrm{b}}$ The value in the absence of peptide was 0 so fold increase in the presence of peptide could not be calculated

No difference in inflammation or meningitis in response to $\mathbf{1 1 0 . 5 8}$ or mutant $\triangle \mathrm{ORF} \mathbf{2}$

Because ORF 2 peptide enhanced growth and AdhE has been linked to inflammation, we tested whether the wild type strain and mutants $\triangle \mathrm{ORF} 1+2$ and $\triangle \mathrm{ORF} 2$ differed in the inflammatory responses they induced in vitro and in vivo. Figure 4 shows that Detroit epithelial cells did not differ in their CXCL8 response to the strains in the presence of $0.07 \mathrm{mg} / \mathrm{ml}$ ORF 2 ligand peptide FPPQSV. For all strains the peptide increased CXCL8 response to the bacteria but we interpret this as being due to increased bacterial growth due to the presence of the peptide. Peptide in the absence of bacteria had no effect on the release of CXCL8 from the Detroit cells as shown in the negative control in Fig. 4.

We also tested whether 110.58 and $\triangle \mathrm{ORF} 1+2$ differed in their ability to cause disease and inflammation in an infant rat model of meningitis. 110.58 was not able to establish a detectable infection in the CSF at $21 \mathrm{~h}$ post infection (hpi) in any rat. For the rats which received the mutant $\triangle$ ORF $1+2$, four had no detectable infection in the CSF at $21 \mathrm{hpi}$, while two had very low levels: 9.5 and $6 \times 10^{4} \mathrm{CFU} / \mathrm{ml}$. No disease, cortical damage, apoptosis in the hippocampus or detection of any of the cytokines

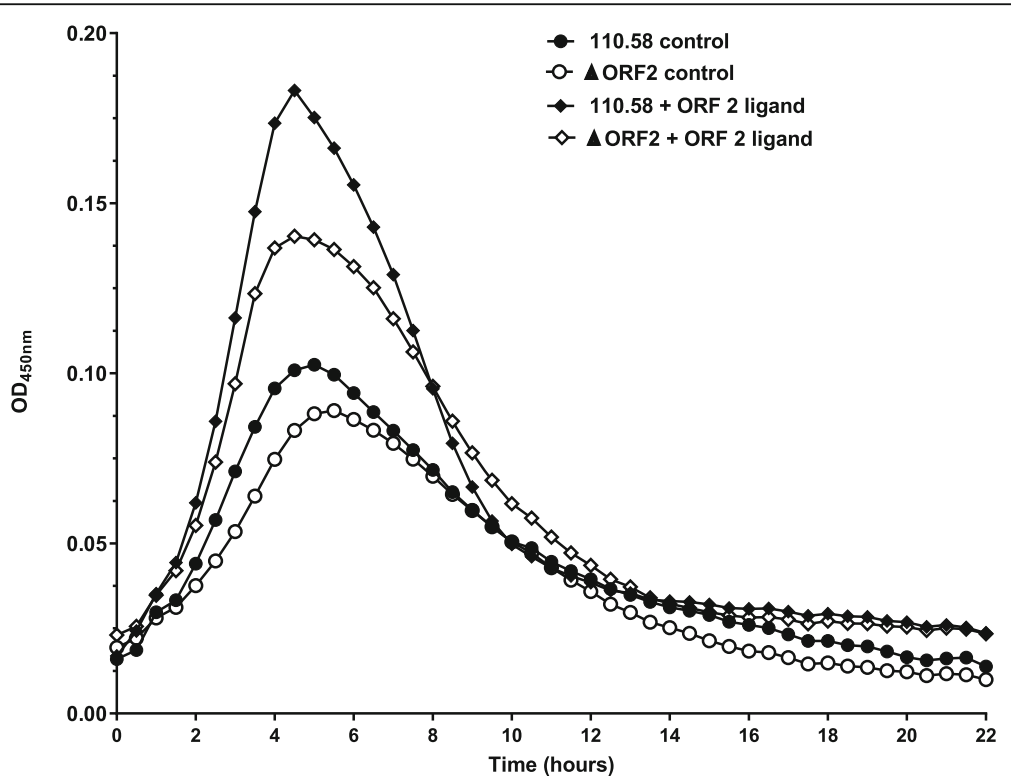

Fig. 2 Growth of wild type strain 110.58 and mutant $\triangle O R F 2$ in CDM with and without $0.062 \mathrm{mg} / \mathrm{ml}$ ORF2 ligand peptide FPPQSV. Curves show the mean values for three independent experiments 

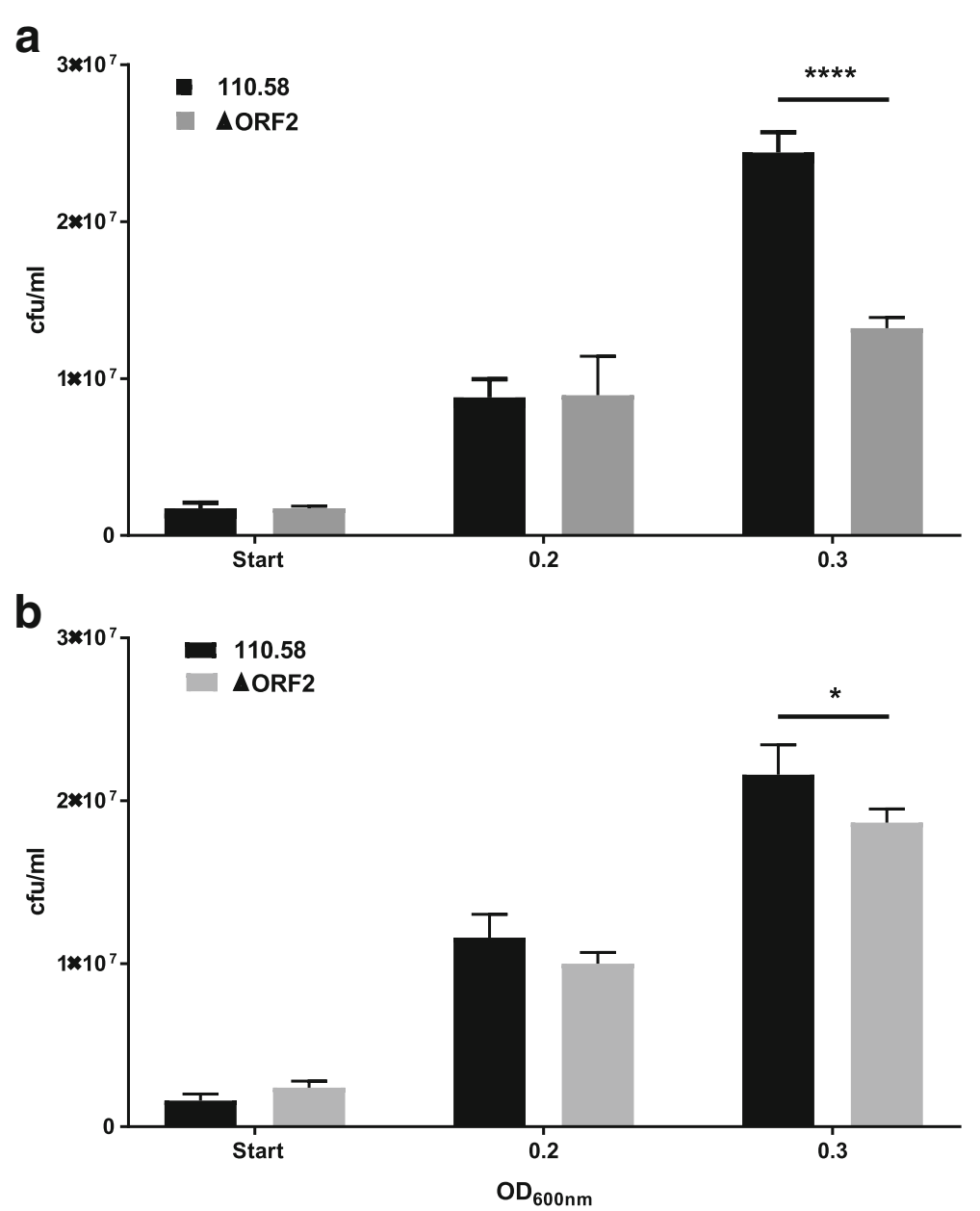

Fig. 3 Competition assay in the presence or absence of ORF 2 peptide. A mixture of wild type strain 110.58 and its mutant $\triangle \mathrm{ORF} 2$ was incubated in (a) the presence of $0.07 \mathrm{mg} / \mathrm{ml}$ of ORF2 ligand peptide FPPQSV or (b) the absence of peptide. At the start and at $\mathrm{OD}_{600 \mathrm{~nm}}=0.2$ and 0.3 $\mathrm{CFU} / \mathrm{ml}$ were determined to show that the wild type has a competitive advantage and that this is greater in the presence of the peptide. ${ }^{* * *} p<0.0001,{ }^{*} p=0.015$. Results are the mean of three independent experiments, error bars show the standard error

IL-6, IL-1 $\beta$, TNF $\alpha$, IL-10, IFN- $\gamma$ in the CSF at 21 or 45 hpi was detected in any rat (data not shown).

\section{Discussion}

Nonencapsulated pneumococci of ST344 which have the genes aliB-like ORF 1 and 2 in place of capsule genes, such as Swiss strain 110.58, are highly successful colonizers of the human nasopharynx, an environment where the concentration of free sugar is considered to be limited [3]. AliB-like ORF 2 appears to aid the early stages of nasopharyngeal colonization, at least in a mouse model [1]. AliB-like ORF 2 can bind peptide FPPQSV, a sequence found in Prevotella species, likely neighbours in the nasopharyngeal niche, indicating a potential route of interspecies communication [1]. For $E$. coli, secreted proteins include ribosomal associated proteins [25] and we speculate that Prevotella too can release proteins usually considered to be intracellular.
Here, we aimed to answer the question of how binding of AliB-like ORF 2 to its ligand may lead to improved colonization. We propose that peptide FPPQSV acts as a specific signal and that following the specific binding, pneumococci respond by upregulating sialidase to produce more free carbohydrate, increasing carbohydrate uptake and increasing carbohydrate metabolism. A permease for the update of AliB-like ORF 2 peptide has not yet been identified but due to the sequence homology of AliB-like ORF 2 to substrate binding protein AliB, after which it is named, we speculate that the Ami-AliA/AliB transporter may be responsible for uptake. This oligopeptide permease has also been proposed to be the route of entry into pneumococcus for Phr peptide of the TprA/PhrA quorum sensing system [26]. TprA/PhrA in strain D39 has also been shown to be activated by PhrA2 which is found in the pneumococcal lineage PMEN1 but not in D39 indicating interstrain gene regulation [27]. 


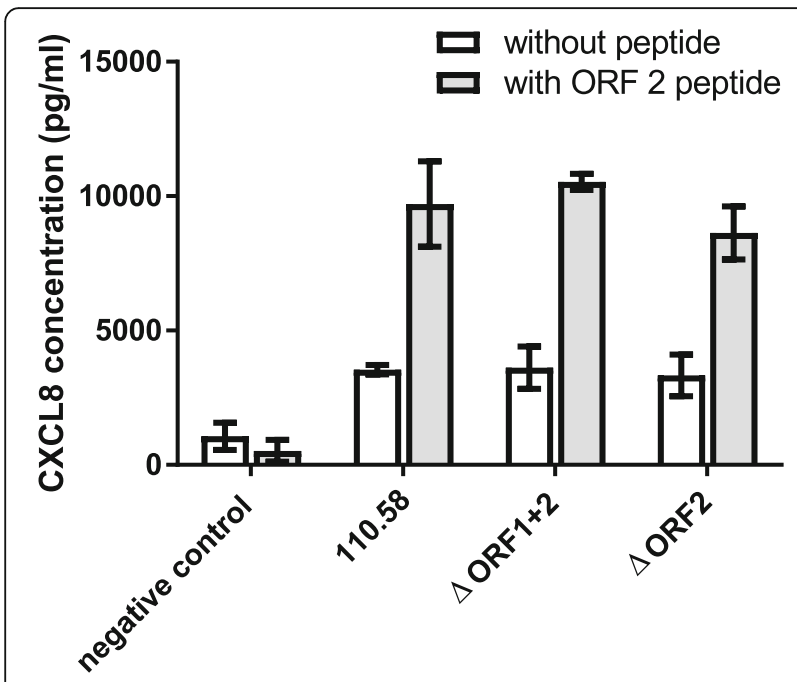

Fig. 4 Effect of wild type pneumococcal strain 110.58 and its mutants $\triangle \mathrm{ORF} 1+2$ and $\triangle \mathrm{ORF} 2$ on CXCL8 induction in human nasopharyngeal Detroit 562 epithelial cells. ORF2 ligand peptide FPPQSV was either not added (open bars) or added to give a final a concentration of $0.07 \mathrm{mg} / \mathrm{ml}$ (shaded bars). No significant differences were observed between the strains under the same conditions. The bars indicate the mean of three independent experiments and error bars indicate standard error. Negative control refers to Detroit cells in the absence of bacteria

Other peptides involved in quorum sensing in pneumococcus include virulence peptide 1 (VP1) which promotes biofilm development on chinchilla middle ear epithelial cells [28] and a small hydrophobic peptide (SHP) which induces capsule expression [29]. However, the crucial difference between these quorum-sensing studies and the work we present here is that we describe the effect of non-streptococcal peptides on pneumococcus i.e. interspecies communication.

In both the transcriptome and proteome data, multiple genes related to carbohydrate metabolism were upregulated by the presence of the ORF 2 peptide ligand. There was a good correlation between these two datasets with afr, encoding 1,5-anhydro-D-fructose reductase, increasing in expression significantly in both when ORF 2 peptide was present. 1,5-anhydro-D-fructose reductase is a metabolic enzyme which acts on 1,5-anhydro-D-fructose, the central intermediate of the anhydrofructose pathway, an alternative starch- and glycogen degrading pathway in bacteria, fungi, plants and mammals [30]. In E. coli the anhydrofructose pathway is proposed to be involved in the stress response related to carbon starvation or reactive oxygen species [31]. Trappetti et al. have also described a link between an interspecies signaling molecule and carbohydrate metabolism in pneumococcus: They found that autoinducer 2 (AI-2), is recognized by FruA (a fructose-specific phosphoenolpyruvatephosphotransferase system) to enable pneumococcus to use galactose, the predominant sugar source in the nasopharynx, as a carbon source [32].

Neuraminidase-encoding genes nanA and nanB both had increased expression in response to ORF 2 peptide. Neuraminidases are thought to cleave sialic acid from cell surface glycans and mucin and both NanA and NanB have been shown to be essential for successful colonization in a mouse model [33]. Exposure of galactose on epithelial cells by NanA promotes biofilm formation during colonization [34]. In addition, deglycosylation of host glycoconjugates by neuraminidases releasing monosaccharides provides $S$. pneumoniae with a source of carbohydrate which can be used to sustain growth [35].

Upregulation of yes $O$ and $y c j$ genes was also noted in the presence of ORF 2 peptide. These are thought to encode components of an ABC transporter for carbohydrate uptake and are found immediately downstream of $n a n B$. Other genes upregulated by ORF 2 peptide believed to be involved in carbohydrate transport and metabolism were sorB_1, $\operatorname{manX} X_{-} 1, \operatorname{agaS}$, agaC_1 and $a d h E$. $\operatorname{tab} A$, proposed to encode a component of a toxin-antitoxin involved in biofilm formation, was also upregulated which is compatible with stepping up colonization. The uracil transporter encoded by pyrP was also of note as this was upregulated in both the RNA and protein data. Cell division protein DivIB was upregulated at the protein level as were RibE and RibH, involved in riboflavin synthesis. Other genes were differentially regulated as well and the strength of gene expression is not necessary related to functional importance. Therefore, it is possible that other pathways that are not discussed here are also involved. In particular, genes belonging to amino acid metabolism and transport were upregulated.

These findings prompted us to look at the effect of ORF 2 peptide on growth. Previously, we had failed to detect a direct effect of the peptide on growth [1] but here we analyzed growth over time in a defined medium devoid of peptides until we added our ORF 2 peptide. This confirmed that the ORF 2 peptide promotes growth. This effect was also seen in the mutant lacking the ORF 2 peptide receptor, although to a lesser extent. This suggests that AliB-like ORF 2 is not the only receptor for the peptide. However, in a competition assay in the presence of the peptide, the wild type strain significantly outcompeted the mutant lacking aliB-like ORF 2 indicating that in nature with multiple strains present, having AliB-like ORF 2 would give an advantage in growth and therefore colonization.

Nonencapsulated strains with aliB-like ORF 1 and 2 are associated with nasopharyngeal colonization [3] and conjunctivitis [36] but not virulent disease. Our results using an in vivo model of meningitis supported this as neither the wild type nor mutant strain caused disease 
when administered intracisternally. This may be due to these nonencapsulated pneumococci being removed by the immune system before any growth advantage of the wild type strain becomes apparent. We also looked at the effect of 110.58 and its mutants on human respiratory cells in vitro and found no difference in their ability to stimulate the epithelial cells to release CXCL8, a marker of an inflammatory response.

\section{Conclusion}

A peptide which matches proteins found in Prevotella species acts on nonencapsulated pneumococci, which have a specific receptor for it, to trigger a boost in carbohydrate metabolism and thus growth. This would seem to be the mechanism by which possession of AliBlike ORF 2 aids colonization. Pneumococci, including virulent encapsulated strains, possess other $A B C$ transporters of oligopeptides which warrant investigation as potential sensors of signals from members of the microbiota which could trigger responses in pneumococci during colonization and disease.

\section{Additional files}

Additional file 1: Figure S1. Venn diagram of RNA-Seq analysis results showing number of genes with different levels of gene expression in untreated wildtype strain 110.58 vs mutant $\triangle$ ORF 2 (purple), wild type with and without ORF 2 ligand peptide FPPQSV (blue) and mutant $\triangle$ ORF 2 with and without peptide FPPQSV (green). The corresponding gene lists, including their expression values, can be found in Additional files 2: Table S1. Additional file 3: Table S2. Additional file 4: Table S3. Additional file 5: Table S4. Figure S2. Network of differentially regulated gene products. Nodes represent genes with significantly different expression in wild type and wild type treated with the peptide ligand (inferred from TIGR4 from STRING database). Edges represent evidence for protein-protein interactions. Proteins belonging to the KEGG pathway "purine metabolism" $\left(\right.$ FDR $\left.3 \times 10^{-5}\right)$ are coloured in blue, KEGG pathway "ribosomal proteins" (FDR $4 \times 10^{-34}$ ) in green, KEGG pathway "alanine, aspartate and glutamate" related genes in lilac, branched-chain amino acid transporter proteins [37] (FDR $4 \times 10^{-34}$ ) in yellow and pathogenesis related genes [38] in red. Other genes are shown in white. Figure S3. Correlation between RNA-Seq and proteome expression data. The Pearson correlation between RNA-Seq and proteomics data across all samples was 0.68 . Figure S4. Growth of wildtype strain 110.58 and mutant $\triangle \mathrm{ORF} 2$ in CDM with and without ORF2 ligand peptide FPPQSV at the concentrations indicated. Curves show the mean values for three independent experiments, error bars indicated SEM. (PDF $658 \mathrm{~kb}$ )

Additional file 2: Table S1. Complete RNA-Seq data. (PDF 7151 kb)

Additional file 3: Table S2. RNA-Seq data for wild type with and without ORF 2 peptide. Table shows only significant changes in expression. A significant change in expression was observed for 210 genes of which 159 were upregulated by the ORF 2 peptide and 51 were downregulated by the peptide. (PDF $209 \mathrm{~kb}$ )

Additional file 4: Table S3. RNA-Seq data for $\triangle \mathrm{ORF} 2$ mutant with and without ORF 2 peptide. Table shows only significant changes in expression. A significant change in expression was observed for 249 genes of which 177 were upregulated by the ORF 2 peptide and 72 were downregulated by the peptide. (PDF $231 \mathrm{~kb}$ )
Additional file 5: Table S4. RNA-Seq data for wild type and $\triangle \mathrm{ORF} 2$ mutant without ORF 2 peptide. Table shows only significant differences in expression. A significant difference in expression was observed for 20 genes of which 6 were more highly expressed in the wildtype and 14 were more highly expressed in the mutant. (PDF $108 \mathrm{~kb}$ )

Additional file 6: Table S5. Complete proteomic data. (PDF $3560 \mathrm{~kb}$ )

Additional file 7: Table S6. Proteomic data for wild type with and without ORF 2 peptide. Table shows only significant changes in expression. A significant change in expression was observed for 12 proteins of which 11 were upregulated by the ORF 2 peptide (also shown in Table 2 in the main text) and 1 was downregulated by the peptide. (PDF $34 \mathrm{~kb}$ )

Additional file 8: Table S7. Proteomic data for $\triangle \mathrm{ORF} 2$ with and without ORF 2 peptide. Table shows only significant changes in expression. A significant change in expression was observed for 22 proteins of which 20 were upregulated by the ORF 2 peptide and 2 were downregulated by the peptide. (PDF $52 \mathrm{~kb}$ )

Additional file 9: Table S8. Proteomic data for wild type and $\triangle \mathrm{ORF} 2$ mutant without ORF 2 peptide. Table shows only significant changes in expression. A significant difference in expression was observed for 19 proteins of which 17 were more highly expressed in the wildtype and 2 were more highly expressed in the mutant. (PDF $46 \mathrm{~kb}$ )

\section{Abbreviations}

adhE: aldehyde-alcohol dehydrogenase; afr: 1,5-anhydro-D-fructose reductase; $\mathrm{BHI}$ : Brain heart infusion broth; $\mathrm{CFU}$ : Colony forming units; CSBA: Columbia sheep blood agar; CSF: Cerebrospinal fluid; FCS: Fetal calf serum; FDR: False discovery rate; hpi: hours post infection; LC-MS/MS: Liquid chromatography-tandem mass spectrometry; MEM: Minimum Essential Medium; PFA: Paraformaldehyde

\section{Acknowledgments}

We thank Suzanne Aebi and Franziska Simon for excellent technical support.

\section{Funding}

This work was supported by grants from the Swiss National Science Foundation, grant number 162808 to L.J.H. and grant number 162583 to S.L.L. and a grant from the Gottfried und Julia Bangerter-Rhyner Stiftung to L.J.H. S.F. received funding from SystemsX.ch. The funding bodies had no role in the design of the study, collection, analysis or interpretation of data or in writing the manuscript.

\section{Availability of data and materials}

The datasets supporting the conclusions of this article are included within the article and its additions files.

\section{Authors' contributions}

LJH conceived the study and drafted the manuscript. FN, SF, DG, SLL and LJH participated in design of the study. FN performed real-time PCR, prepared samples for proteomics analysis and performed growth and competition assays. SF prepared samples for RNA-Seq and did all computational analyses concerning RNA-Seq data and the comparison with proteomic data. EY and DG determined disease potential and analyzed host response. $\mathrm{MH}$ processed the proteomics data and performed the label-free protein quantification. All authors were involved in data interpretation and gave final approval for publication.

\section{Ethics approval}

All animal studies were approved by the Animal Care and Experimentation Committee of the Canton of Bern, Switzerland (licence BE76/14) and followed the Swiss national guidelines for the performance of animal experiments.

\section{Consent for publication}

Not applicable.

\section{Competing interests}

The authors declare that they have no competing interests. 


\section{Publisher's Note}

Springer Nature remains neutral with regard to jurisdictional claims in published maps and institutional affiliations.

\section{Author details}

'Institute for Infectious Diseases, Faculty of Medicine, University of Bern, Friedbühlstrasse 51, CH-3001 Bern, Switzerland. ${ }^{2}$ Graduate School for Cellular and Biomedical Sciences, University of Bern, Bern, Switzerland. ${ }^{3}$ Institute of Social and Preventive Medicine, University of Bern, Bern, Switzerland. ${ }^{4}$ Proteomics and Mass Spectrometry Core Facility, Department for BioMedical Research, University of Bern, $\mathrm{CH}-3010$ Bern, Switzerland.

Received: 23 August 2017 Accepted: 15 March 2018 Published online: 27 March 2018

\section{References}

1. Hathaway L, Bättig P, Reber S, Rotzetter J, Aebi S, Hauser C, Heller M, Kadioglu A, Mühlemann K. Streptococcus pneumoniae detects and responds to foreign bacterial peptide fragments in its environment. Open Biol. 2014:4:130224.

2. Hathaway L, Meier PS, Battig P, Aebi S, Muhlemann K. A homologue of aliB is found in the capsule region of nonencapsulated Streptococcus pneumoniae. J Bacteriol. 2004;186:3721-9.

3. Hilty M, Wüthrich $D$, Salter $S$, Engel H, Campbell $S$, Sa-Leao R, Hd L, Hermans $P$, Sadowy $E$, Turner $P$, et al. Global phylogenomic analysis of nonencapsulated Streptococcus pneumoniae reveals a deep-branching classic lineage that is distinct from multiple sporadic lineages. Genome Biology and Evolution. 2014;6:3281-94.

4. Hanage W, Kaijalainen T, Saukkoriipi A, Rickcord J, Spratt B. A successful, diverse disease-associated lineage of nontypeable pneumococci that has lost the capsular biosynthesis locus. J Clin Microbiol. 2006:44:743-9.

5. Croucher N, Coupland P, Stevenson A, Callendrello A, Bentley S, Hanage W. Diversification of bacterial genome content through distinct mechanisms over different timescales. Nat Commun. 2014;5:5471.

6. Valentino M, McGuire A, Rosch J, Bispo P, Burnham C, Sanfilippo C, Carter R, Zegans M, Beall B, Earl A, et al. Unencapsulated Streptococcus pneumoniae from conjunctivitis encode variant traits and belong to a distinct phylogenetic cluster. Nat Commun. 2014;5:5411.

7. Denapaite D, Bruckner R, Nuhn M, Reichmann P, Henrich B, Maurer $P$, Schahle Y, Selbmann P, Zimmermann W, Wambutt R, et al. The genome of Streptococcus mitis B6 - what is a commensal? PLoS One. 2010;5:e9426.

8. Reichmann P, Nuhn M, Denapaite D, Bruckner R, Henrich B, Maurer P, Rieger M, Klages S, Reinhard R, Hakenbeck R. Genome of Streptococcus oralis strain Uo5. J Bact. 2011:193:2888-9.

9. Shahinas D, Tamber G, Arya G, Wong A, Lau R, Jamieson F, Ma J, Alexander D, Low D, Pillai D. Whole-genome sequence of Streptococcus pseudopneumoniae isolate IS7493. J Bact. 2011;193:6102-3.

10. Schaffner T, Hinds J, Gould K, Wüthrich D, Bruggmann R, Küffer M, Mühlemann K, Hilty M, Hathaway L. A point mutation in cpsE renders Streptococcus pneumoniae nonencapsulated and enhances its growth, adherence and competence. BMC Microbiol. 2014;14:210-22.

11. Hathaway L, Brugger S, Morand B, Bangert M, Rotzetter J, Hauser C, Graber W, Gore S, Kadioglu A, Muhlemann K. Capsule type of Streptococcus pneumoniae determines growth phenotype. PLoS Pathog. 2012;8:e1002574.

12. Brewster J. A simple micro-growth assay for enumerating bacteria. J Microbiol Methods. 2003:53:77-86.

13. Hathaway L, Battig P, Muhlemann K. In vitro expression of the first capsule gene of Streptococcus pneumoniae, cpsA, is associated with serotype-specific colonization prevalence and invasiveness. Microbiology. 2007;153:2465-71.

14. Bolger A, Lohse M, Usadel B. Trimmomatic: a flexible trimmer for Illumina sequence data. Bioinformatics. 2014:30:2114-20.

15. Langmead B, Salzberg S. Fast gapped-read alignmet with bowtie 2 . Nat Methods. 2012:9:357-9.

16. Trapnell C, Roberts A, Goff L, Pertea G, Kim D, Kelley D, Pimentel H, Salzberg S, Rinn J, Pachter L. Differential gene and transcript expression analysis of RNA-seq experiments with TopHat and cufflinks. Nat Protoc. 2012;7:562-78.

17. Okonechnikov K, Conesa A, García-Alcalde F. Qualimap 2: advanced multisample quality control for high-throughput sequencing data. Bioinformatics. 2016:32:292-4.
18. Engel H, Mika M, Denapaite D, Hakenbeck R, Mühlemann K, Heller M, Hathaway L, Hilty M. A low-affinity penicillin-binding protein $2 x$ variant is required for heteroresistance in Streptococcus pneumoniae. Antimicrob Agents Chemother. 2014:58:3934-41.

19. Lazar C, Gatto L, MF M, Bruley C, Burger T. Accounting for the multiple natures of missing values in label-free quantitative proteomics data sets to compare imputation strategies. J Proteome Res. 2016;15:1116-25.

20. Baumgartner D, Aebi S, Grandgirard D, Leib S, Draeger A, Babiychuk E, Hathaway L. Clinical Streptococcus pneumoniae isolates induce differing CXCL8 responses from human nasopharyngeal epithelial cells which are reduced by liposomes. BMC Microbiol. 2016;16:154.

21. Liechti F, Grandgirard D, Leppert D, Leib S. Matrix metalloproteinase inhibition lowers mortality and brain injury in experimental pneumococcal meningitis. Infect Immun. 2014;82:1710-8.

22. Hathaway L, Grandgirard D, Valente L, Täuber M, Leib S. Streptococcus pneumoniae capsule determines disease severity in experimental pneumococcal meningitis. Open Biol. 2016;6:150269.

23. Liechti F, Grandgirard D, Leib S. The antidepressant fluoxetine protects the hippocampus from brain damage in experimental pneumococcal meningitis. Neuroscience. 2015;297:89-94.

24. Luong T, Kim E, Bak J, Nguyen C, Choi S, Briles D, Pyo S, Rhee D. Ethanolinduced alcohol dehydrogenase $\mathrm{E}$ (AdhE) potentiates pneumolysin in Streptococcus pneumoniae. Infect Immun. 2015;83:108-19.

25. Lee E-Y, Bang JY, Park GW, Choi D-S, Kang JS, Kim H-J, Park K-S, Lee J-O, Kim $\mathrm{Y}-\mathrm{K}$, Kwon K-H, et al. Global proteomic profiling of native outer membrane vesicles derived from Escherichia coli. Proteomics. 2007:7:3143-53.

26. Hoover S, Perez A, Tsui H, Sinha D, Smiley D, DiMarchi R, Winkler M, Lazazzera B. A new quorum sensing system (TprA/PhrA) for Streptococcus pneumoniae D39 that regulates a lantibiotic biosynthesis gene cluster. Mol Microbiol. 2015;97:229-43.

27. Kadam A, Eutsey R, Rosch J, Miao X, Longwell M, Xu W, Woolford C, Hillman T, Motib A, Yesilkaya $\mathrm{H}$, et al. Promiscuous signaling by a regulatory system unique to the pandemic PMEN1 pneumococcal lineage. PLoS Pathog. 2017:13

28. Cuevas R, Eutsey R, Kadam A, West-Roberts J, Woolford C, Mitchell A, Mason K, Hiller N. A novel streptococcal cell-cell communication peptide promotes pneumococcal virulence and biofilm formation. Mol Microbiol. 2017;105:554-71.

29. Junges R, Salvadori G, Shekhar S, Amdal H, Periselneris J, Chen T, Brown J, Petersen F. A quorum-sensing system that regulates Streptococcus pneumoniae biofilm formation and surface polysaccharide production. mSphere. 2017;2

30. Schu M, Faust A, Stosik B, Kohring G, Giffhorn F, Scheidig A. The structure of substrate-free 1,5-anhydro-D-fructose reductase from Sinorhizobium meliloti 1021 reveals an open enzyme conformation. Acta Crystallogr Sect F Struct Biol Cryst Commun. 2013:69:844-9.

31. Yu S. The anhydrofructose pathway of glycogen catabolism. IUBMB Life. 2008:60:798-809.

32. Trappetti C, McAllister L, Chen A, Wang H, Paton A, Oggioni M, McDevitt C, Paton J. Autoinducer 2 signaling via the phosphotransferase FruA drives galactose utilization by Streptococcus pneumoniae, resulting in Hypervirulence. MBio. 2017:8:e02269-16.

33. Manco S, Hernon F, Yesilkaya H, Paton J, Andrew P, Kadioglu A. Pneumococcal neuraminidases a and $B$ both have essential roles during infection of the respiratory tract and sepsis. Infect Immun. 2006;74:4014-20.

34. Blanchette K, Shenoy A, Milner J, Gilley R, McClure E, Hinojosa C, Kumar N, Daugherty S, Tallon L, Ott S, et al. Neuraminidase A-exposed galactose promotes Streptococcus pneumoniae biofilm formation during colonization. Infect Immun. 2016:84:2922-32.

35. Burnaugh A, Frantz L, King S. Growth of Streptococcus pneumoniae on human glycoconjugates is dependent upon the sequential activity of bacterial exoglycosidases. J Bacteriol. 2008;190:221-30.

36. Martin M, Turco J, Zegans M, Facklam R, Sodha S, Elliott J, Pryor J, Beall B, Erdmann D, Baumgartner $Y$, et al. An outbreak of conjunctivitis due to atypical Streptococcus pneumoniae. N Engl J Med. 2003;348:1112-21.

37. Basavanna S, Khandavilli S, Yuste J, Cohen J, Hosie A, Webb A, Thomas G, Brown J. Screening of Streptococcus pneumoniae ABC transporter mutants demonstrates that LivJHMGF, a branched-chain amino acid $A B C$ transporter, is necessary for disease pathogenesis. Infect Immun. 2009;77:3412-23.

38. Kadioglu A, Weiser J, Paton J, Andrew P. The role of Streptococcus pneumoniae virulence factors in host respiratory colonization and disease. Nat Rev Microbiol. 2008;6:288-301. 